International Journal of Mechanical Engineering and Technology (IJMET)

Volume 12, Issue 2, February 2021, pp. 35-40. Article ID: IJMET_12_02_004

Available online at https://iaeme.com/Home/issue/IJMET?Volume $=12 \&$ Issue $=2$

ISSN Print: 0976-6340 and ISSN Online: 0976-6359

DOI: https://doi.org/10.34218/IJMET.12.2.2021.004

(c) IAEME Publication

Scopus Indexed

\title{
ESTIMATING THE VELOCITY DISTRIBUTION IN VISCOUS INCOMPRESSIBLE FLOW (COUETTE FLOW) BETWEEN TWO PARALLEL PLATES USING TRI -DIAGONAL MATRIX ALGORITHM
}

\author{
Rakesh Sudarsi
}

\begin{abstract}
Numerical solutions to Viscous Incompressible flow between two parallel plates have been developed over the last half century. Multi-level techniques are successfully developed and applied for the same problem where significant speed-ups are achieved. Due to complexity of Navier-Stokes equation, obtaining analytical solutions for viscous flow problems if difficult sometimes. Obtaining exact solution of Navier-Stokes equation is possible for some classical cases of steady, laminar, viscous and incompressible flows. Numerical research in this area will help researchers to develop techniques which yield accurate and faster solutions. The present work is on estimating the velocity distribution in Couette flow between two parallel plates by using Newtonian fluids as lubricant. The resulting partial differential equations of Navier-Stokes equation after the boundary conditions are substituted are solved by Finite Difference Method. An algorithm named as "Tri Diagonal Matrix Algorithm" is generated for the same. By using MATLAB, numerical analysis is performed to obtain the velocity distribution between two parallel plates.
\end{abstract}

Keywords: Couette flowk, Tri Diagonal Matrix Algorithm.

Cite this Article: Rakesh Sudarsi, Estimating the Velocity Distribution in Viscous Incompressible Flow (Couette Flow) Between Two Parallel Plates Using Tri -Diagonal Matrix Algorithm, International Journal of Mechanical Engineering and Technology (IJMET), 12(2), 2021, pp.35-40.

https://iaeme.com/Home/issue/IJMET?Volume=12\&Issue $=2$

\section{INTRODUCTION}

Obtaining analytical solutions for viscous flow problems is practically difficult. The NavierStokes equations are the basic differential equations which describe the flow of incompressible Newtonian fluids. Navier-Stokes equations are non-linear and second order partial differential equations. The non-linear nature of convective acceleration terms in Navier-Stokes equation 
are difficult to handle in a physical situation. Moreover, there are no general analytical schemes for solving the non-linear partial differential equations. Due to the nature of the geometry of the flow system, convective acceleration vanish in some applications. So, the exact solutions are possible. The Navier-Stokes equations are applicable to both laminar and turbulent flows. Due to fluctuations in velocity components for turbulent flow, these exact solutions are referred to laminar flows. For a laminar flow, the velocity is independent of time (Steady flow) or dependent on time (unsteady flow). When viscous incompressible flow between two flat plates with no pressure gradient is considered, where the upper plate moves with constant velocity and the lower plate is fixed, the velocity distribution across the channel is linear with Newtonian fluid as lubricant [1]. A typical application of couette flow is found in the journal bearing where the main crankshaft rotates with an angular velocity and the outer housing is stationary. So, the present work will serve as the base for studying the tribological properties of a journal bearing.

\section{COUETTE FLOW BETWEEN TWO PARALLEL PLATES WITH NEWTONIAN FLUID AS LUBRICANT}

Consider two-dimensional incompressible, viscous, laminar flow between two parallel plates which are $2 \mathrm{~h}$ distance apart. The upper plate moves with constant velocity "V" while the lower is fixed. For the flow to be axial $(u \neq 0, v=0, w=0)$ the plates are assumed to be very wide and long. There is no pressure gradient [2].

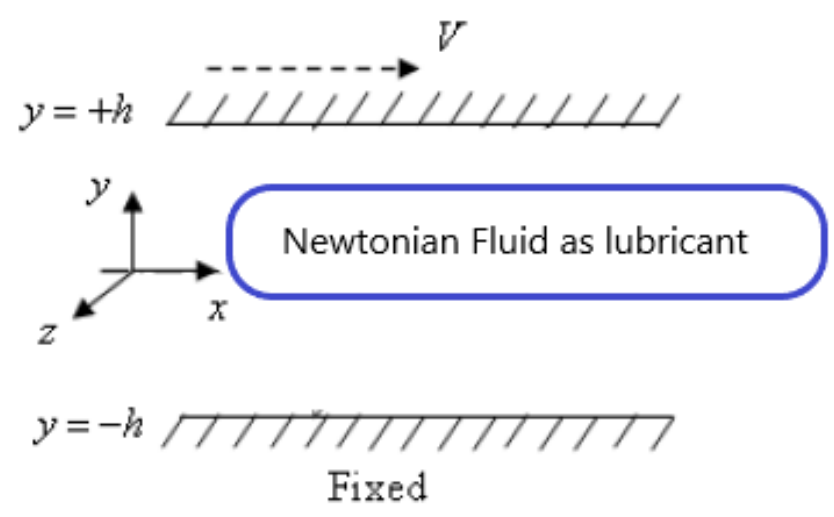

Neglecting gravity effects, the continuity equation for incompressible flow can be written as:

$$
\frac{\partial U}{\partial X}+\frac{\partial V}{\partial Y}+\frac{\partial W}{\partial Z}=0
$$

As $\mathbf{V}=\mathbf{W}=\mathbf{0}$, the continuity equation reduces to

$$
\frac{\partial U}{\partial X}=\mathbf{O}
$$

which means $\mathbf{U}=\mathbf{U}(\mathbf{Y})$ only

This indicates that the velocity component will vary only across the channel [2]. So, the $\mathrm{X}$ component of Navier - Stokes momentum equation is considered.

$$
\rho\left(U \frac{\partial U}{\partial X}+V \frac{\partial U}{\partial Y}\right)=-\frac{\partial p}{\partial X}+\rho g_{X}+\mu\left(\frac{\partial^{2} U}{\partial X^{2}}+\frac{\partial^{2} u}{\partial Y^{2}}\right)
$$

From (5)

$\mathbf{U}=\mathbf{U}(\mathbf{Y})$ which implies

$$
\frac{\partial U}{\partial X}=\frac{\partial^{2} U}{\partial X^{2}}=\mathbf{0}
$$


No pressure gradient i.e

$$
\frac{\partial \mathbf{p}}{\partial \mathbf{x}}=\mathbf{0}
$$

As gravity always act in vertically downward, $\mathbf{g}_{\mathbf{x}}=\mathbf{0}$

Substituting all these in the momentum equation (6)

$$
\frac{\partial^{2} U}{\partial Y^{2}}=0
$$

This is the derivation for viscous incompressible flow between two flat plates from the basics of fluid mechanics [3]

\section{FINITE DIFFERENCE APPROXIMATION FOR PARTIAL DIFFERENTIAL EQUATIONS}

Derivatives in the partial differential equation are approximated by the linear combinations of function values at the grid points.

Consider a rectangular domain $\mathrm{D}: 0 \leq \mathrm{X} \leq \mathrm{a}$ and $0 \leq \mathrm{Y} \leq \mathrm{b}$

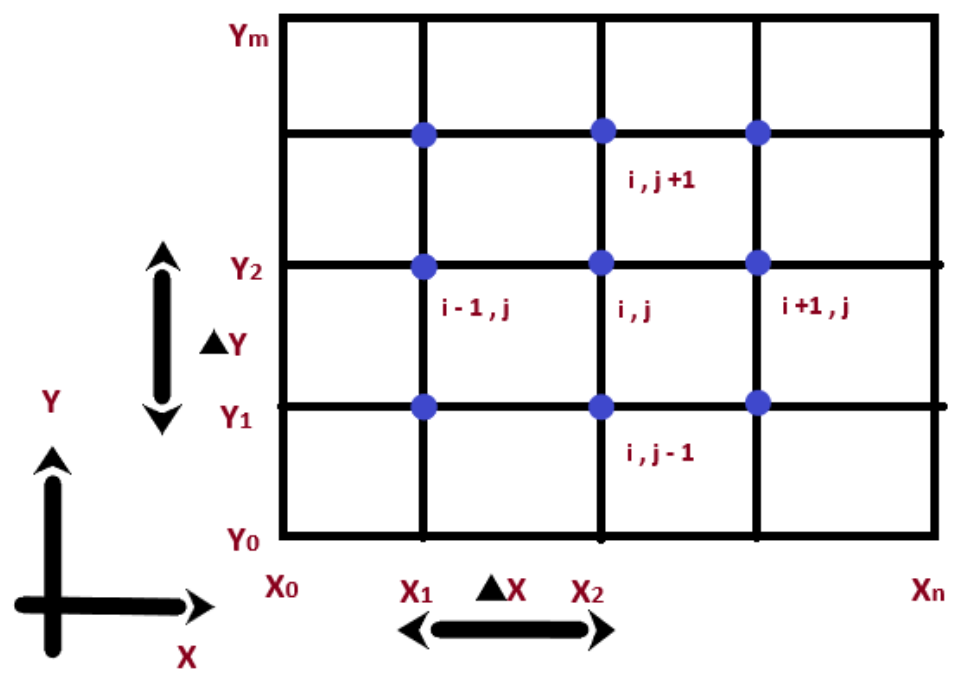

Figure 1

Draw straight lines parallel to $\mathrm{X}$-axis and $\mathrm{Y}$-axis as shown in the figure above such that

$$
\begin{aligned}
& X_{i}=i * \Delta X(i=1,2,3----n-1) \\
& Y_{j}=j * \Delta Y(j=1,2,3-----m-1)
\end{aligned}
$$

Where $\Delta \mathrm{X}$ and $\Delta \mathrm{Y}$ are small positive step lengths

$$
\begin{aligned}
& \Delta X=\frac{a}{n} \\
& \Delta Y=\frac{b}{m}
\end{aligned}
$$

If $P_{i j}=P\left(X_{i}, Y_{j}\right)$ be any point in the region $\mathrm{D}$ then the coordinates $X_{i}$ and $Y_{j}$ can be obtained by

$$
\begin{gathered}
X_{i}=X_{0}+i * \Delta X \\
Y_{i}=Y_{0}+j * \Delta Y
\end{gathered}
$$

Where $\left(X_{0}, Y_{0}\right)$ are the coordinates of the left bottom most point of the rectangle grid considered. 
Estimating the Velocity Distribution in Viscous Incompressible Flow (Couette Flow) Between Two Parallel Plates Using Tri -Diagonal Matrix Algorithm

If $\mathrm{U}(\mathrm{X}, \mathrm{Y})$ is any continuous function with all necessary derivatives existing in $\mathrm{D}$ [4] then

$$
\begin{gathered}
U_{i \pm 1, j}=U(X \pm \Delta X, Y)=U_{i, j} \pm \Delta X \frac{\partial U_{i, j}}{\partial X} \pm \frac{\Delta X^{2}}{2 !} \frac{\partial^{2} U_{i, j}}{\partial X^{2}} \pm-------- \\
U_{i, j \pm 1}=U(X, Y \pm \Delta Y)=U_{i, j} \pm \Delta Y \frac{\partial U_{i, j}}{\partial Y} \pm \frac{\Delta Y^{2}}{2 !} \frac{\partial^{2} U_{i, j}}{\partial Y^{2}} \pm---------
\end{gathered}
$$

First order Partial derivatives in (13) can be approximated as [5]

Central Difference:

$$
\frac{\partial U_{i, j}}{\partial Y}=\frac{U_{i, j+1}-U_{i, j-1}}{2 \Delta Y}
$$

Forward Difference:

$$
\frac{\partial U_{i, j}}{\partial Y}=\frac{U_{i, j+1}-U_{i, j}}{\Delta Y}
$$

Backward difference:

$$
\frac{\partial U_{i, j}}{\partial Y}=\frac{U_{i, j+1}-U_{i, j}}{\Delta Y}
$$

The second order partial derivatives in expression (13) can be approximated as

$\frac{\partial^{2} U_{i, j}}{\partial Y^{2}}=\frac{U_{i, j+1}-2 U_{i, j}+U_{i, j-1}}{\Delta Y^{2}}$

Making use of these approximations to replace partial derivatives, the partial differential equations are converted into difference equations and the resultant system of algebraic equations are solved using iterative methods.

\section{TRI DIAGONAL MATRIX ALGORITHM FOR THE COUETTE FLOW PROBLEM}

The reduced Navier-Stokes momentum equation for a 2D viscous incompressible flow (Couette flow) between two parallel plates from (7) is

$$
\frac{\partial^{2} \mathbf{U}}{\partial \mathbf{Y}^{2}}=0
$$

From (14), the above partial differential equation can be substituted by

$$
U_{i, j+1}-2 U_{i, j}+U_{i, j-1}=0
$$

$$
\frac{\partial^{2} U_{i, j}}{\partial Y^{2}}=\frac{U_{i, j+1}-2 U_{i, j}+U_{i, j-1}}{\Delta Y^{2}}=0
$$

The height between the two plates is divided into N nodes. $(i=1 ; j=1$ to $N$ )

Boundary conditions:

At the lower fixed plate: when $\mathbf{i}=\mathbf{1} ; \mathbf{j}=\mathbf{1}$ then $\mathbf{U}=\mathbf{0}$

At the upper moving plate: when $\mathbf{i}=\mathbf{1} ; \mathbf{j}=\mathbf{N}$ then $\mathbf{U}=\mathbf{V}$

Substituting the values of $i$ and $j$ in (15)

$\mathrm{i}=1 ; \mathrm{j}=1$

$\mathrm{i}=1 ; \mathrm{j}=2$

$$
U_{1,2}-2 U_{1,1}+U_{1,0}=0
$$

$\mathrm{i}=1 ; \mathrm{j}=3$

$$
U_{1,3}-2 U_{1,2}+U_{1,1}=0
$$

$$
U_{1,4}-2 U_{1,3}+U_{1,2}=0
$$

$\mathrm{i}=1 ; \mathrm{j}=4$ 
Rakesh Sudarsi

$$
\begin{array}{lc}
\mathrm{i}=1 ; \mathrm{j}=5 & U_{1,5}-2 U_{1,4}+U_{1,3}=0 \\
- & U_{1,6}-2 U_{1,5}+U_{1,4}=0 \\
- & \\
- & \\
\mathrm{i}=1 ; \mathrm{j}=\mathrm{N}-1 & \\
\mathrm{i}=1 ; \mathrm{j}=\mathrm{N} & U_{1, N}-2 U_{1, N-1}+U_{1, N-2}=0 \\
& U_{1, N+1}-2 U_{1, N}+U_{1, N-1}=0
\end{array}
$$

The above equations are written in matrix form as shown below

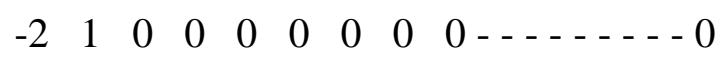

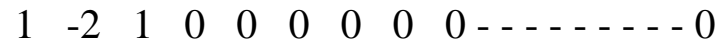

$\begin{array}{lllllllllllllllll}0 & 1 & -2 & 1 & 0 & 0 & 0 & 0 & 0 & \ldots & \ldots & \ldots & \ldots\end{array}$

$\begin{array}{lllllllllllllll}0 & 0 & 1 & -2 & 1 & 0 & 0 & 0 & 0 & \ldots & \ldots & \ldots & \ldots\end{array}$

$\mathrm{x}$

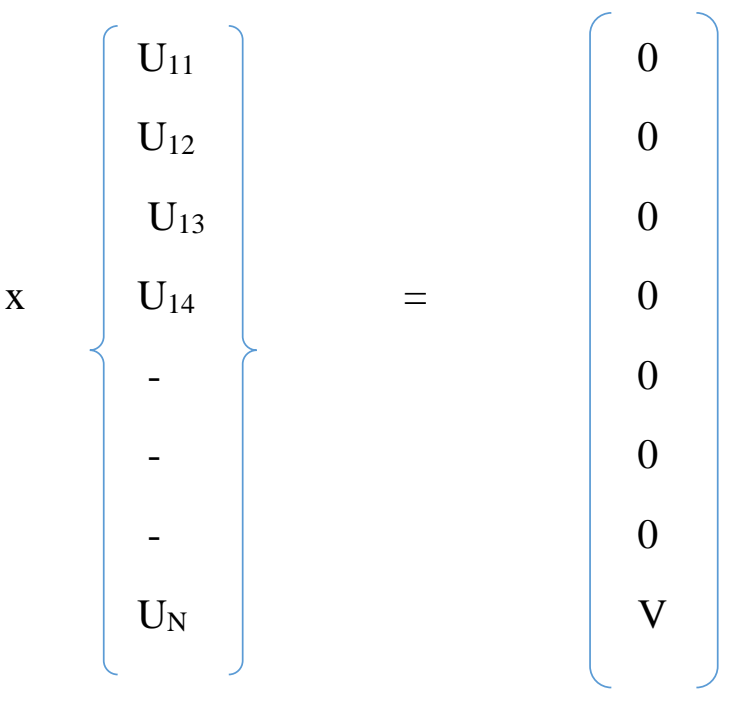

\section{RESULTS}

Let velocity of the upper plate $=10 \mathrm{~m} / \mathrm{s}$

Height between the plates is divided into $10 \operatorname{nodes}(\mathrm{j}=0$ to 1$)$

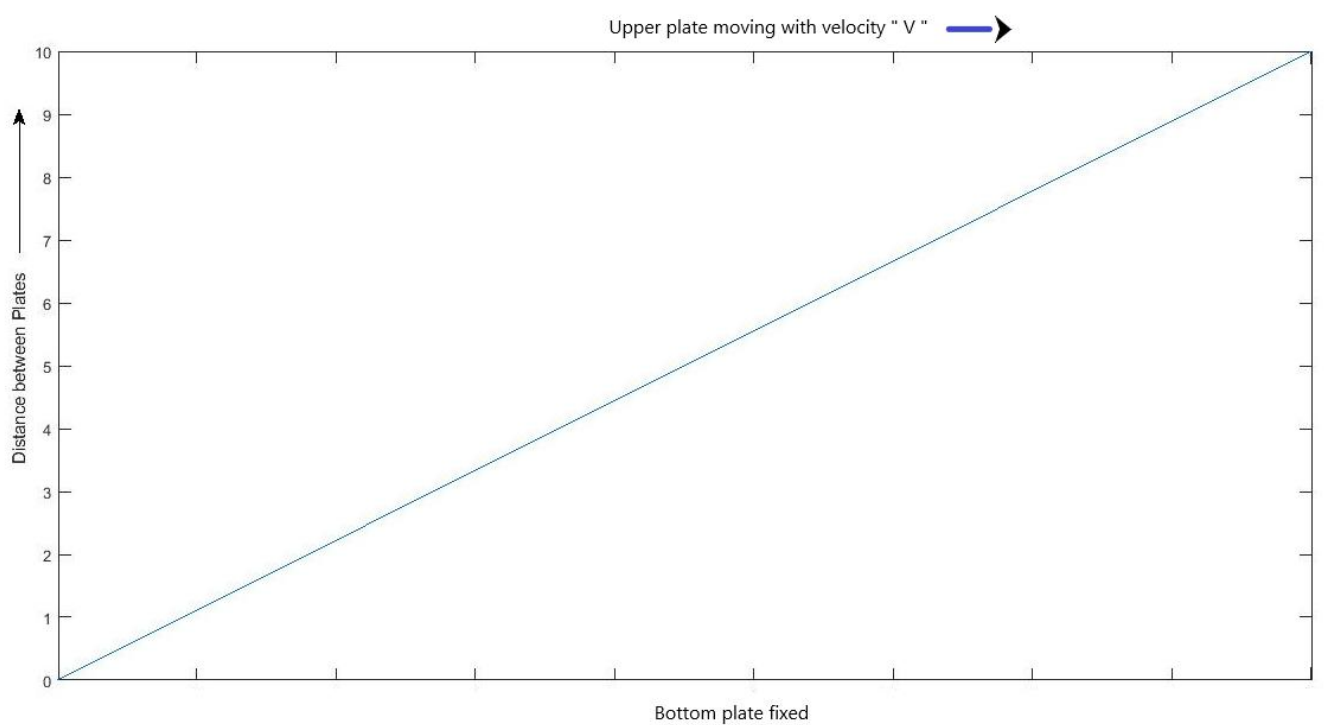

Figure 2 
Estimating the Velocity Distribution in Viscous Incompressible Flow (Couette Flow) Between Two Parallel Plates Using Tri -Diagonal Matrix Algorithm

By using MATLAB, the coefficients of finite difference equations which are in TriDiagonal matrix form are plotted to get the velocity distribution for viscous incompressible flow between two parallel plates. It can be observed from the graph that the velocity distribution is linear for a couette flow between two parallel plates. The Newton's law of viscosity for Newtonian fluids is satisfied.

\section{CONCLUSIONS}

- The velocity profile for couette flow between two parallel plates with Newtonian fluid is linear

- The velocity obtained will vary only across the channel, $U=U$ (Y)

- Couette flow between two parallel plates can be analysed by replacing Newtonian fluid with Non - Newtonian fluids by which comparison can be made between the two fluids for further study and research.

\section{REFERENCES}

[1] John.J.Bloomer, Practical fluid Mechanics for Engineering Applications (New York, Marcel Dekker, 2000)

[2] Joseph H.Spurk, Nuri Aksel, Fluid Mechanics $2^{\text {nd }}$ edition (Germany: Springer, 2008)

[3] Frank.M.White, Fluid Mechanics, $8^{\text {th }}$ edition (New York: Tata Mc Graw Hill, 2016)

[4] John Noye, Finite Difference Techniques for Partial Differential Equations, Computational techniques for Differential equations, North Holland Mathematics Studies (Holland: Elsevier,1984)

[5] J.W.Thomas, Numerical Partial Differential Equations: Finite Difference Methods (New York: Springer, 1995) 